\title{
On the Asymptotic Convergence of Collocation Methods With Spline Functions of Even Degree
}

\author{
By J. Saranen and W. L. Wendland*
}

\begin{abstract}
We investigate the collocation of linear one-dimensional strongly elliptic integro-differential or, more generally, pseudo-differential equations on closed curves by even-degree polynomial splines. The equations are collocated at the respective midpoints subject to uniform nodal grids of the even-degree $B$-splines. We prove quasioptimal and optimal order asymptotic error estimates in a scale of Sobolev spaces. The results apply, in particular, to boundary element methods used for numerical computations in engineering applications. The equations considered include Fredholm integral equations of the second and the first kind, singular integral equations involving Cauchy kernels, and integro-differential equations having convolutional or constant coefficient principal parts, respectively.

The error analysis is based on an equivalence between the collocation and certain variational methods with different degree splines as trial and as test functions. We further need to restrict our operators essentially to pseudo-differential operators having convolutional principal part. This allows an explicit Fourier analysis of our operators as well as of the spline spaces in terms of trigonometric polynomials providing Babuška's stability condition based on strong ellipticity.

Our asymptotic error estimates extend partly those obtained by D. N. Arnold and W. L. Wendland from the case of odd-degree splines to the case of even-degree splines.
\end{abstract}

1. Introduction. In this paper we investigate the asymptotic convergence of the collocation method using even-degree polynomial splines applied to strongly elliptic systems of pseudo-differential equations on closed curves with convolutional principal part. The collocation here employs the Gauss points of one-point integration as collocation points which corresponds to the usual boundary element collocation in applications [5], [12], [17], [19], [26], [31], [34]. This is in contrast to the method investigated by G. Schmidt in [29] where one collocates at the break points of even-order splines.

The asymptotic convergence properties for the standard Galerkin method with splines of arbitrary orders are well-known [22]. For the collocation method, however, asymptotic convergence, up to now, has been shown for strongly elliptic systems only in the case of odd-order splines by D. N. Arnold and W. L. Wendland [7].

Received November 22, 1982; revised May 2, 1984.

1980 Mathematics Subject Classification. Primary 65R20; Secondary 65L10, 65N35, 45J05, 45F15, 35S99, 30C30, 73K 30.

*This work was carried out while the first author was an "Alexander von Humboldt-Stiftung" research fellow at the Technical University of Darmstadt during the academic year 1981-82.

The second author wants to express his gratitude to Professor Dr. C. Johnson and the Department of Applied Mathematics and Computer Science since the discussions during his visit in 1982 at Chalmers University have been very stimulating for our results, in particular in connection with (3.19). 
Collocation with even-order splines at the Gauss points has been investigated only for Fredholm integral equations of the second kind [27]. Here we investigate the much wider class of strongly elliptic systems and we show asymptotic convergence of optimal order for even-order spline collocation.

These approximations are widely used in engineering numerical analysis as boundary element methods for two-dimensional stationary or time-harmonic interior and exterior boundary value problems as well as transmission problems. The corresponding boundary integral operators belong to various different classes. However, the Fourier transform became an appropriate tool and now the theory of pseudo-differential operators provides the framework for a unifying mathematical analysis. Since this modern part of analysis is rather new, it is not yet general knowledge, although it provides the common basic properties of differential as well as integral operators including the whole variety arising in the boundary-value problems of many engineering applications.

Our convergence results assure, in particular, optimal asymptotic convergence for piecewise constant spline collocation for equations of negative order as, e.g., Symm's integral equation of the first kind [31] and its generalizations [15], [19], [20], [21], [26], [34], [35]. For this equation preliminary convergence results can be found in [1], [2], [5], [14] and [33]. Our strongly elliptic systems with convolutional principal part contain, in addition, systems of integro-differential equations [3] (see [7]) with constant coefficients, certain singular integral equations, in particular, those of plane elasticity [7, Appendix], [24], [25], [26], [34], Fredholm integral equations of the second kind [6], [8], [11], [12], [13], [17], [27], [35], and also the integro-differential operator of Prandtl's wing theory [16], [17], [18], [24], [35].

Our rigorous error estimates cover the case of even-order splines left open in [7] for the above subclass of strongly elliptic equations. Our analysis is based on the reformulation of the collocation equations as equivalent Galerkin-Petrov equations with different splines as test and trial functions. In order to secure the Babuška stability conditions with the help of strong ellipticity, we now need to assign an appropriate test spline of degree $d+1$ to any choice of trial spline of degree $d$. The construction of this mapping $Q$ was suggested by the ellipticity condition in connection with the finitely Fourier transformed splines.

The general pseudo-differential operators on closed curves have a particularly simple action on Fourier series [4], [28]. Therefore, we shall restrict our formulation to the corresponding manipulations with the Fourier coefficients, avoiding the close connections to the general theory. In this case our assumptions can be formulated without the theory of pseudo-differential operators solely in terms of the action on Fourier series, and the inexperienced reader can start with property (2.10) and Section 3. However, the theory for elliptic pseudo-differential operators is behind the ideas of our proofs and will be decisive for an extension of the convergence results from the present case of constant coefficients to the more general equations involving variable coefficients.

Our analysis is performed in the space of Fourier series; we first analyze the Fourier series of the spline functions finding periodic recurrence relations for their Fourier coefficients. This analysis, with an appropriate choice of spline bases interpolating trigonometric polynomials, allows us to define the above-mentioned mapping $Q$, which provides, in combination with strong ellipticity, the Babuška 
stability conditions. The latter yields optimal-order convergence in the associated energy norm. In addition, we apply the Aubin-Nitsche duality arguments as in [7] to obtain super approximations. For simplicity, we develop this analysis first for just one equation and then extend our results to systems in Section 3.2.

The authors want to thank Prof. Dr. D. N. Arnold and the referee of the first version for their helpful remarks.

2. Applications. The range of applications of our result already covers a relatively large set of integro-differential equations, singular integral equations, etc., acting on functions which are defined on closed smooth curves in the two-dimensional space. Our convergence proof is based on an explicit Fourier representation (formula (2.10)) of the operators in question, which is assumed in this work, and whose validity for pseudo-differential operators is given in [4] and [28] (Theorem 2.1). In most applications this representation can also be obtained by direct computations. For classical pseudo-differential operators, only the principal symbol $\sigma(\xi)$ [30], [32] needs to be known in order to have the Fourier representation. Here, our main results are formulated without referring to pseudo-differential operators. But because of the above-mentioned connection we mention the main types of applications and for our analysis we need to write out the corresponding principal symbols.

The equations to be discussed are of the form

$$
A u+B \omega=f, \quad \Lambda u=\beta,
$$

where the vector-valued function $u=\left(u_{1}, \ldots, u_{p}\right)$ and the vector $\omega \in \mathbf{C}^{q}$ denote desired unknowns, and $f=\left(f_{1}, \ldots, f_{p}\right)$, as well as $\beta \in \mathbf{C}^{q}$, are given. Moreover, $A$ is a system of integro-differential operators or singular integral operators or, in general, pseudo-differential operators, respectively. $B$ and $\Lambda$ are matrices of appropriate functions or functionals, respectively, $B: \mathbf{C}^{q} \rightarrow\left(H^{t_{1}}\right)^{p}$ and $\Lambda:\left(H^{t_{2}}\right)^{p} \rightarrow$ $\mathrm{C}^{q}$, where $H^{t_{i}}$ denote Sobolev spaces to be specified later on; see [35].

For the convergence of the collocation method, our main assumption for the operator $A$ will be strong ellipticity and that the principal part is a pure convolution. In the framework of pseudo-differential operators this means that the principal symbol $\sigma=\sigma(\xi)$ depends only on the Fourier transformed variable $\xi$, and that there exist a regular complex-valued $p \times p$ matrix $\Theta$ and a constant $\sigma_{0}>0$, such that

$$
\operatorname{Re} \zeta^{\mathrm{T}} \Theta \sigma(\xi) \bar{\zeta} \geqslant \sigma_{0}|\zeta|^{2} \quad \text { for } \xi= \pm 1 \text { and } \zeta \in \mathbf{C}^{p}
$$

Moreover, $\sigma(\xi)$ is a positive-homogeneous function of degree $2 \alpha$.

This class of equations (2.1) contains the following more special examples; see [7, Section 2.3]. (There, one also finds references to corresponding applications.)

2.1. Integro-Differential Equations With Cauchy Kernel and Constant Coefficient Principal Part. These equations are of the form

$$
\begin{gathered}
A_{0} \frac{d^{m} u}{d s^{m}}+\sum_{j=0}^{m-1} A_{m-j}(s) \frac{d^{j} u}{d s^{j}} \\
+\frac{1}{\pi i} \int_{\Gamma}\left\{C_{0} \frac{d^{m} u}{d \tau^{m}}(\tau)+\sum_{j=0}^{m-1} C_{m-j}(s, \tau) \frac{d^{j} u}{d \tau^{j}}(\tau)\right\} \frac{d \zeta}{\zeta-z(s)} \\
+\int_{\Gamma} L(s, \tau) u(\tau) d \tau+\sum_{k=1}^{q} \omega_{k} B_{k}(s)=f(s), \\
\Lambda u=\beta, \quad m \in \mathbf{N}_{0},
\end{gathered}
$$


where $\zeta=z_{1}(\tau)+i z_{2}(\tau), A_{0}, C_{0}$ are constant $p \times p$ matrices, and where $s$, respectively $\tau$, denotes the arclength on $\Gamma$. The principal symbol of this operator is given by the matrix-valued function [3]

$$
\sigma(\xi)=i^{m} \xi^{m}\left(A_{0}+C_{0} \frac{\xi}{|\xi|}\right) \text { for }|\xi| \geqslant 1 .
$$

2.1.1. The Case of Even $m$. In the case when $m$ is even, $\alpha=m / 2$ is a nonnegative integer and the strong ellipticity condition (2.2) now reads

$$
(-1)^{\alpha} \operatorname{Re} \zeta^{\mathrm{T}} \Theta\left(A_{0} \pm C_{0}\right) \bar{\zeta} \geqslant \sigma_{0}|\zeta|^{2},
$$

which must hold for both signs and is equivalent to

$$
\operatorname{det}\left(A_{0}+\lambda C_{0}\right) \neq 0 \text { for all } \lambda \in[-1,+1] .
$$

Note that this condition precludes the possibility that det $A_{0}=0$.

In the special case $C \equiv 0$ and $L \equiv 0$ the operator in (2.3) reduces to a system of ordinary differential operators of even order $2 \alpha$. The ellipticity condition is definiteness of the leading coefficient matrix $A_{0}$, and our results provide new error estimates for the collocation of periodic ordinary differential equations with periodic side conditions by even-degree splines provided the principal part of the differential equations has constant coefficients.

Singular integral equations. In the case $\alpha=m=0,(2.3)$ reduces to a system of singular integral equations with Cauchy kernel. Up to now for these equations only collocation by splines of odd degree has been investigated [7]. But note that $G$. Schmidt [29] proves optimal-order convergence results with even-degree spline collocation at the break points for Eqs. (2.3) if

$$
\operatorname{det}\left(\lambda A_{0}+C_{0}\right) \neq 0 \text { for all } \lambda \in[-1,1],
$$

which is a somewhat complementary class of equations to ours.

Singular integral equations with constant principal coefficients satisfying (2.4) occur, e.g., in plane elasticity [7, (2.3.7)], [24], [25], [26], [34].

Fredholm integral equations of the second kind. A further specialization of (2.3) to the case $m=\alpha=0, A_{0}=I, C_{0} \equiv 0$, yields the Fredholm integral equations of the second kind

$$
u(s)+\int_{\Gamma} L(s, \tau) u(\tau) d \tau+B(s) \omega=f(s), \quad \Lambda u=\beta
$$

The principal symbol $\sigma$ is here the identity matrix, so the equations are trivially strongly elliptic and our results apply. However, they can be obtained in this case from well-known results [6], [13] in combination with approximation estimates (3.25) as in [27]. For the numerical treatment and further convergence results see [8] and [11].

Our error estimates apply, in particular, to the method in [12] for $C^{1}$-piecewise quadratic splines (i.e., third-order splines), if $\Gamma$ is smooth and either the Dirichlet or the Neumann problem is solved for the Fredholm integral equations of the second kind in acoustics (see [35] and references given there). 
2.1.2. The Case of Odd $m$. Here, let us recall the presentation in [7] and turn to systems involving operators of the form (2.3) with odd order $m$. Then $\alpha-\frac{1}{2}=$ $(m-1) / 2$ is a nonnegative integer and instead of (2.4) the strong ellipticity condition is

$$
(-1)^{\alpha-1 / 2} \operatorname{Re} i \zeta^{\mathrm{T}} \Theta\left( \pm A_{0}+C_{0}\right) \bar{\zeta} \geqslant \sigma_{0}|\zeta|^{2}
$$

which again must hold for both signs. Condition (2.5) is equivalent to

$$
\operatorname{det}\left(\lambda A_{0}+C_{0}\right) \neq 0, \quad \lambda \in[-1,1] .
$$

Hence, in this case of odd order $m$, det $C_{0}$ cannot vanish.

An example is given by the normal derivative of the double-layer potential, that is, the operator defined by

$$
\begin{aligned}
\tilde{C}_{0} u(s) & =\frac{1}{\pi} \frac{\partial}{\partial \nu_{z}} \int_{\Gamma} u(\tau) \frac{\partial}{\partial \nu_{\zeta}}(\log |z-\zeta|) d \tau \\
& =\frac{1}{\pi} \int_{\Gamma}(u(\tau)-u(s)) \frac{\partial}{\partial \nu_{z}} \frac{\partial}{\partial \nu_{\zeta}}(\log |z-\zeta|) d \tau
\end{aligned}
$$

$z=z(s)$ and $\zeta=z(\tau)$ on $\Gamma$, which plays an important role in classical potential theory. Equations of the form

$$
\begin{gathered}
A_{1}(s) u(s)+\tilde{C}_{0} u(s)+\frac{1}{\pi i} \int_{\Gamma} C_{1}(s, \tau) u(\tau) \frac{d \zeta}{\zeta-z(s)} \\
+\int_{\Gamma} L(s, \tau) u(\tau) d \tau+B \omega=f(s), \\
\Lambda u=\beta,
\end{gathered}
$$

$\zeta=z(\tau)$ on $\Gamma$, have been used more recently in acoustics and electromagnetic fields and corresponding numerical treatments, [16], [17], [18], [35]. Employing the Cauchy-Riemann equations and integration by parts, (2.6) can be rewritten as

$$
\tilde{C}_{0} u=-\operatorname{Re} \frac{1}{\pi} \int_{\Gamma} \frac{d u}{d \tau}(\tau)\left(\frac{d z}{d s}(s) \overline{\frac{d z}{d \tau}(\tau)}\right) \frac{d \zeta}{\zeta-z(s)}
$$

whose principal part is given by

$$
-\frac{1}{i \pi} \int_{\Gamma} \frac{d u}{d \tau}(\tau) \frac{i d \zeta}{\zeta-z(s)}
$$

which is the famous Prandtl integro-differential operator of wing theory; [24] and [25]. From (2.8) we see that (2.7) is a specialization of (2.3) to the case $A_{0}=0$, $C_{0}=-i, m=1$ and the principal symbol is $|\xi|$. Hence (2.7) is strongly elliptic and $\Theta$ in (2.5) can be taken to be the identity.

2.2. Fredholm Integral Equations of the First Kind With Logarithmic Principal Part. A large class of interior and exterior boundary-value problems in two dimensions can be reduced to systems of the form

$$
-\frac{1}{\pi} \int_{\Gamma}(\log |z(s)-z(\tau)|+L(s, \tau)) u(\tau) d \tau+B(z(s)) \omega=f(z(s))
$$

$$
\int_{\Gamma} \Lambda(\tau) u(\tau) d \tau=\beta
$$


where the kernel $L$ is smoother than the logarithmic principal part. For this operator the order is $2 \alpha=-1$, and the principal symbol is $\sigma(\xi)=|\xi|^{-1} I$, so the operator is strongly elliptic (and $\Theta=I$ ). Applications with (2.9) can be found in potential theory [1], [19], [20], [31], flow problems [15], [20], [21], acoustics [17], [34] and elasticity [7], [26], [34].

Our convergence results assure for the first time the convergence of the numerical methods in [31], where piecewise constant trial functions have been used, and in [19] and [26], where piecewise quadratic splines have been used for (2.9).

2.3. Fourier Series Representation. Let

$$
z=\left(z_{1}(\theta), z_{2}(\theta)\right) \simeq z_{1}(\theta)+i z_{2}(\theta)
$$

be a regular parametric representation of $\Gamma$, where $z$ is a 1-periodic function of the real variable $\theta$ with $|d z / d \theta| \neq 0$. Then every function on $\Gamma$ can be identified with its 1-periodic image depending on $\theta$. More generally, for a system of mutually disjoint Jordan curves $\Gamma=\bigcup_{j=1}^{L} \Gamma_{j}$ we may parametrize each of them and identify functions on $\Gamma$ with $L$ vector-valued 1-periodic functions of $\theta$. Hence, without loss of generality, we may consider systems (2.1), where all functions, respectively, distributions, are 1-periodic depending on $\theta$.

Since all 1-periodic distributions can be represented in the form of Fourier series

$$
u=\sum_{n \in \mathbf{Z}} \hat{u}_{n} e^{i n 2 \pi \theta}, \quad \theta \in \mathbf{R},
$$

we shall now characterize the class of operators to which our results apply by means of the Fourier representation. Simple examples of such operators are given by the Hilbert transform defined by the Cauchy principal-value integral

$$
H u:=\text { p.v. } \int_{0}^{1} \frac{u(\phi) d \phi}{1-e^{2 \pi i(\theta-\phi)}}=\sum_{n \in \mathbf{Z}} \hat{u}_{n} \operatorname{sign}(n) e^{i n 2 \pi \theta}
$$

where

$$
\operatorname{sign}(n)=\left\{\begin{array}{cc}
+1 & \text { for } n \geqslant 0, \\
-1 & \text { for } n<0,
\end{array}\right.
$$

which has the principal symbol $\sigma(H, \xi)=\xi /|\xi|$ and by the simple layer potential

$$
V u(\theta):=2 \int_{0}^{1} \log \left|e^{i 2 \pi \theta}-e^{i 2 \pi \phi}\right| u(\phi) d \phi=\sum_{n \neq 0} \frac{1}{|n|} u_{n} e^{i n 2 \pi \theta}
$$

which has the principal symbol $\sigma(V, \xi)=1 /|\xi|,[20]$.

We define the operators

$$
P_{2 \alpha}^{+} u:=\sum_{n>0} n^{2 \alpha} \hat{u}_{n} e^{i n 2 \pi \theta}+\hat{u}_{0} \quad \text { and } \quad P_{2 \alpha}^{-} u:=\sum_{n<0}|n|^{2 \alpha} \hat{u}_{n} e^{i n 2 \pi \theta}
$$

In the following, $H^{s}$ denotes the periodic Sobolev space of arbitrary real order $s$, i.e., the closure of all smooth 1-periodic functions with respect to the norm

$$
\|f\|_{s}:=\|f\|_{H^{\prime}}:=\left(\left|\hat{f}_{0}\right|^{2}+\sum_{0 \neq n \in \mathbf{Z}} \hat{f}_{n} \cdot \overline{\hat{f}}_{n}|2 \pi n|^{2 s}\right)^{1 / 2},
$$

where $\hat{f}_{n}$ are the Fourier coefficients. We denote the inner product in this space by

$$
\langle f, g\rangle_{s}:=\hat{f}_{0} \cdot \overline{\hat{g}}_{0}+\sum_{0 \neq n \in \mathbf{Z}} \hat{f}_{n} \cdot \overline{\hat{g}}_{n}|2 \pi n|^{2 s} \text {. }
$$


Note that this inner product extends to a duality pairing between $H^{s+\alpha}$ and $H^{s-\alpha}$ for arbitrary real $\alpha$, and, moreover,

$$
\sup _{n \in H^{-\alpha}} \frac{\left|\langle v, w\rangle_{s}\right|}{\|w\|_{s-\alpha}}=\|v\|_{s+\alpha}, \quad v \in H^{s+\alpha} .
$$

Clearly, $H^{s}$ is continuously imbedded into $H^{t}$ for $s>t$ (see [23, p. 37]).

In the following our crucial assumption on the operator $A: H^{s} \rightarrow H^{s-2 \alpha}$ will be that it has the decomposition

$$
A=\sigma_{+} P_{2 \alpha}^{+}+\sigma_{-} P_{2 \alpha}^{-}+K
$$

where $K: H^{s} \rightarrow H^{s-2 \alpha}$ is compact. Clearly, the operators $H$ and $V$ already have the form (2.10). Similarly, the integro-differential equations (2.3) take the form (2.10),

$$
\begin{aligned}
A_{0} \frac{d^{m} u}{d \theta^{m}}(2 \pi i)^{-m} & +C_{0} H\left[\frac{d^{m} u}{d \phi^{m}}(2 \pi i)^{-m}\right]+K_{1} u \\
& =\left(A_{0}+C_{0}\right) P_{m}^{+} u+(-1)^{m}\left(A_{0}-C_{0}\right) P_{m}^{-} u+K_{2} u
\end{aligned}
$$

after multiplication by $|d z(\theta) / d \theta|(2 \pi i)^{-m}$, as do the equations of the form (2.9) after multiplication by $2 \pi /|d z / d \theta|$, since

$$
-\frac{1}{\pi} \int_{\Gamma} \log |z(s)-z(\tau)| u d \tau \frac{2 \pi}{|d z / d \theta|}=V u+K_{3} u,
$$

where $K$, denote compact operators.

From the point of view of the general pseudo-differential operators on the unit circle we can use the following result [4], [28].

THEOREM 2.1. Let $A: H^{s} \rightarrow H^{s-2 \alpha}$ be a pseudo-differential operator with a positivehomogeneous principal symbol of real order $2 \alpha$. Then $A$ has the representation

$$
A=\sigma_{+} P_{2 \alpha}^{+}+\sigma_{-} P_{2 \alpha}^{-}+K, \quad \sigma_{+}=\sigma(1), \quad \sigma_{-}=\sigma(-1),
$$

where $K: H^{s} \rightarrow H^{s-2 \alpha}$ is a compact operator.

Of course, Theorem 2.1 applies also to systems of pseudo-differential operators of the common order $2 \alpha$.

\section{The Convergence of the Collocation Method.}

3.1. Single Equations Without Side Conditions. For simplicity we consider first the special case of (2.1) of just one equation with $B, \Lambda$ and $\beta$ all $=0$ and $A$ a given operator of the form (2.10). Hence, we shall investigate the approximation of the equation

$$
A u=f
$$

by the standard collocation method. To this end, we select an increasing sequence of mesh points

$$
x_{j}=j \frac{1}{N}=j h \quad \text { with } j=0,1, \ldots, N ; \quad \Delta=\left\{x_{j}\right\},
$$

with $N$ running through the natural numbers.

In addition, we introduce the nodal points

$$
t_{j}=\frac{j-1 / 2}{N}=\left(j-\frac{1}{2}\right) h, \quad j=1, \ldots, N ; \quad \tilde{\Delta}=\left\{t_{j}\right\} .
$$


By $S_{d}(\Delta)$ we denote the space of all 1-periodic, $d-1$ times continuously differentiable splines of degree $d$ subordinate to the partition $\Delta$ if $d \geqslant 1$. By $S_{0}(\Delta)$ we denote the corresponding step functions. Correspondingly, we shall also need $S_{\tilde{d}}(\tilde{\Delta})$ with $\tilde{d}=d+1$.

The collocation method for (3.1) is:

Find $u_{\Delta} \in S_{d}(\Delta)$ such that the collocation equations

$$
A u_{\Delta}\left(t_{j}\right)=f\left(t_{j}\right), \quad j=1, \ldots, N,
$$

are satisfied.

Since $S_{d}(\Delta) \subset H^{d+1 / 2-\varepsilon}$ with any $\varepsilon>0, A u_{\Delta}$ will be continuous under the assumption

$$
d>2 \alpha .
$$

(Although this condition seems to be rather natural, it often is too restrictive for practical computations.)

For our error analysis we shall now write the collocation equations in the form of modified Petrov-Galerkin equations with different test and trial functions. Theorem 2.1.1 in [7] yields:

Lemma 3.1. Let $d \geqslant 0$ be an even integer and let $u \in H^{j+\alpha-1 / 2}$. Then the collocation equations (3.2) are satisfied if and only if

$$
\left\langle\left(I-J+J_{\tilde{\Delta}}\right) A u_{\Delta}, \phi\right\rangle_{j}=\left\langle\left(I-J+J_{\tilde{\Delta}}\right) A u, \phi\right\rangle_{j} \text { for all } \phi \in S_{d+1}(\tilde{\Delta}),
$$

where $j=d / 2+1$ and

$$
J u:=\int_{0}^{1} u(x) d x \text { and } J_{\tilde{\Delta}} u:=\sum_{l=1}^{N} h u\left(t_{l}\right) .
$$

Clearly, the equations (3.4) are modified equations of a Galerkin-Petrov method to find $w_{\Delta} \in S_{d}(\Delta)$ such that

$$
\left\langle A w_{\Delta}, \phi\right\rangle_{j}=\langle A u, \phi\rangle_{j} \text { for all } \phi \in S_{d+1}(\tilde{\Delta}) .
$$

Since $S_{d}(\Delta) \subset H^{d+1 / 2-\varepsilon}$ for any $\varepsilon>0$, we have with $\varepsilon=d / 2-\alpha$ viz. (3.3), that $S_{d}(\Delta) \subset H^{j+\alpha-1 / 2}$. Correspondingly, $S_{d+1}(\tilde{\Delta}) \subset H^{j+\alpha-1 / 2}$. Therefore, the sesquilinear form (3.5) is continuous on $H^{j+\alpha-1 / 2} \times H^{j+\alpha+1 / 2}$.

The following theorem provides stability of the method (3.5).

THEOREM 3.2. Let $A$ have the special form

$$
A=\sigma_{+} P_{2 \alpha}^{+}+\sigma_{-} P_{2 \alpha}^{-} \text {with } \operatorname{Re} \sigma_{+}>0, \operatorname{Re} \sigma_{-}>0,
$$

i.e., $A$ is strongly elliptic. Then there exists a positive constant $\gamma$ such that the Babuška stability condition

$$
\inf _{\substack{l, \in S_{d}(\Delta) \\\|v \cdot\|_{j+\alpha-1 / 2}=1}} \sup _{\substack{\phi \in S_{d+1}(\tilde{\Delta}) \\\|\phi\|_{j+\alpha+1 / 2}=1}}\left|\langle A v, \phi\rangle_{j}\right| \geqslant \gamma
$$

is satisfied.

Proof. For $S_{d}(\Delta)$ and $S_{d+1}(\tilde{\Delta})$ we shall use the special spline bases as defined in [9, Chapter 4]. Let $\pi$ be the characteristic function of $[0,1)$ and let

$$
\pi^{d+1}:=\pi * \pi * \cdots * \pi=(* \pi)^{d+1}
$$


denote the $d$-fold convolution of $\pi$. Then the $B$-splines

$$
v_{j}^{d}(t)=\left\{\begin{array}{l}
\pi^{d+1}(t / h-j+1) \text { for }(j-1) h \leqslant t<(j-1) h+1 \\
\text { and the 2-periodic extension, } j=1, \ldots, N,
\end{array}\right.
$$

form a basis of $S_{d}(\Delta)$ and, correspondingly,

$$
\phi_{j}^{d+1}(t):=v_{j}^{d+1}\left(t+\frac{h}{2}\right), \quad j=1, \ldots, N,
$$

form a basis of $S_{d+1}(\tilde{\Delta})$.

In order to prove the assertions (3.6) we shall investigate the Fourier coefficients of the splines. The combination of the convolutions in (3.7) with Fourier transformation provides us with the property

$$
\left(\hat{v}_{h}^{d}\right)_{n+N}=\left(\frac{n}{n+N}\right)^{d+1}\left(\hat{v}_{j}^{d}\right)_{n} \text { for } n \neq 0 \text { modulo } N
$$

Therefore, we find for any natural $l$ and any $v \in S_{d}(\Delta)$ that

$$
(\hat{v})_{n+I N}=\left(\frac{n}{n+I N}\right)^{d+1}(\hat{v})_{n} \text { for } n \neq 0 \text { modulo } N \text {. }
$$

For $0 \neq n=l N$ we find $\left(\hat{v}_{j}^{d}\right)_{n}=0$ and, accordingly,

$$
(\hat{v})_{n}=0 \text { for } 0 \neq n=l N .
$$

Besides $v_{j}^{d}$, we introduce a new basis in $S_{d}(\Delta)$ and $S_{d+1}(\tilde{\Delta})$, respectively, by using the finite Fourier transform. Specifically, we define

$$
\begin{aligned}
w_{k}^{d}(t) & =\sum_{j=1}^{N} e^{i k 2 \pi t_{j}} v_{j}^{d}(t), \quad k=0,1, \ldots, N-1, \\
\tilde{w}_{k}^{d+1}(t) & =\sum_{j=1}^{N} e^{i k 2 \pi t} \phi_{j}^{d+1}(t), \quad k=0,1, \ldots, N-1 .
\end{aligned}
$$

Then $\left\{w_{k}^{d}\right\}_{0}^{N-1}$ is a basis of $S_{d}(\Delta)$ and $\left\{\tilde{w}_{k}^{d+1}\right\}_{0}^{N-1}$ is a basis of $S_{d+1}(\tilde{\Delta})$. We have

$$
\tilde{w}_{k}^{d+1}(t)=\left(\pi_{h} * w_{k}^{d}\right)\left(t+\frac{h}{2}\right)
$$

where $\pi_{h}(t)=h^{-1} \pi\left(h^{-1} t\right)$.

For the Fourier coefficients of these new basis functions we calculate

$$
\begin{aligned}
\left(\hat{\tilde{w}}_{k}^{d+1}\right)_{n} & =e^{i n \pi / N}\left(\hat{\pi}_{h}\right)_{n}\left(\hat{w}_{k}^{d}\right)_{n} \\
& = \begin{cases}\frac{N}{n \pi} \sin \left(\frac{n \pi}{N}\right)\left(\hat{w}_{k}^{d}\right)_{n} & \text { for } n \neq 0 \text { and } \\
\left(\hat{w}_{k}^{d}\right)_{0} & \text { for } n=0 .\end{cases}
\end{aligned}
$$

Moreover, we obtain by (3.9) and by

$$
\left(\hat{v}_{j}^{d}\right)_{n}=e^{i 2 \pi n(1-j) / N}\left(\hat{v}_{1}^{d}\right)_{n}
$$

with an elementary computation the recurrence relation

$$
\begin{aligned}
\left(\hat{w}_{k}^{d}\right)_{n} & =\left\{\sum_{j=1}^{N}\left[e^{i(k-n) 2 \pi / N}\right]^{j}\right\} e^{i(n 2 \pi / N-k \pi / N)}\left(\hat{v}_{1}^{d}\right)_{n} \\
& = \begin{cases}0 & \text { for } k-n \neq l N, l \in \mathbf{Z}, \\
N\left(\hat{v}_{1}^{d}\right)_{n} e^{i(n 2 \pi / N-k \pi / N)} & \text { for } n=k+l N .\end{cases}
\end{aligned}
$$


In particular, if for $n \in \mathbf{Z}$ we denote by $[n]$ the unique integer $[n] \in\{0,1, \ldots$, $N-1\}$ such that $n=[n]+l N$ for some $l \in \mathbf{Z}$, then the above relation can be written as

$$
\left(\hat{w}_{k}^{d}\right)_{n}=\delta_{k[n]}\left(\hat{w}_{[n]}^{d}\right)_{n} .
$$

Accordingly, for any $v \in S_{d}(\Delta)$ with

$$
v=\sum_{k=0}^{N-1} c_{k} w_{k}^{d}
$$

we find for the Fourier coefficients

$$
(\hat{v})_{n}=c_{[n]}\left(\hat{w}_{[n]}^{d}\right)_{n} .
$$

Correspondingly, if

$$
\phi=\sum_{k=0}^{N-1} d_{k} \tilde{w}_{k}^{d+1} \in S_{d+1}(\tilde{\Delta}),
$$

we obtain from (3.10) and (3.11), the relation

$$
(\hat{\phi})_{n}= \begin{cases}\frac{N}{n \pi} \sin \frac{n \pi}{N} d_{[n]}\left(\hat{w}_{[n]}^{d}\right)_{n} & \text { for } n \neq 0 \\ d_{0} & \text { for } n=0\end{cases}
$$

With $v$ in the form (3.11) we have, by (3.12), the explicit representation

$$
\begin{aligned}
(A v)(t)= & \sigma_{+} c_{0}+\sigma_{+} \sum_{n>0} n^{2 \alpha} c_{[n]}\left(\hat{w}_{[n]}^{d}\right)_{n} e^{i n 2 \pi t} \\
& +\sigma_{-} \sum_{n<0}|n|^{2 \alpha} c_{[n]}\left(\hat{w}_{[n]}^{d}\right)_{n} e^{i n 2 \pi t} .
\end{aligned}
$$

Thus, we have for the bilinear form

$$
\begin{aligned}
\langle A v, \phi\rangle_{j}= & \sigma_{+} c_{0} \bar{d}_{0}+(2 \pi)^{2 j} \sigma_{+} \sum_{n>0} n^{2(\alpha+j)} \frac{N}{n \pi} \sin \frac{n \pi}{N} c_{[n]} \bar{d}_{[n]}\left|\left(\hat{w}_{[n]}^{d}\right)_{n}\right|^{2} \\
& +(2 \pi)^{2 j} \sigma_{-} \sum_{n<0}|n|^{2(\alpha+j)} \frac{N}{n \pi} \sin \frac{n \pi}{N} c_{[n]} \bar{d}_{[n]}\left|\left(\hat{w}_{[n]}^{d}\right)_{n}\right|^{2}
\end{aligned}
$$

In order to show the inequalities (3.6), we choose for any $v \in S_{d}(\Delta)$ the test function $\phi \in S_{d+1}(\tilde{\Delta})$ by taking

$$
d_{k}= \begin{cases}\frac{1}{2 N} \frac{1}{|\sin k \pi / N|} c_{k} & \text { for } k=1, \ldots, N-1, \\ c_{0} & \text { for } k=0 .\end{cases}
$$

With this choice of $\phi$ we have, by (3.15),

$$
\begin{aligned}
\langle A v, \phi\rangle_{j}= & \sigma_{+}\left|c_{0}\right|^{2}+(2 \pi)^{2 j-1} \sigma_{+} \sum_{\substack{n>0 \\
n \neq m N}} n^{2(\alpha+j)-1} \varepsilon(n)\left|c_{[n]}\left(\hat{w}_{[n]}^{d}\right)_{n}\right|^{2} \\
& +(2 \pi)^{2 j-1} \sigma_{-} \sum_{\substack{n>0 \\
n \neq m N}} n^{2(\alpha+j)-1} \varepsilon(n)\left|c_{[-n]}\left(\hat{w}_{[-n]}^{d}\right)_{-n}\right|^{2} \\
= & : \sigma_{+}\left|c_{0}\right|^{2}+(2 \pi)^{2 j-1}\left(\sigma_{+} \Sigma^{+}+\sigma_{-} \Sigma^{-}\right)
\end{aligned}
$$


where

$$
\varepsilon(n)= \begin{cases}1 & \text { for } 0<n \leqslant N-1 \text { modulo } 2 N \\ -1 & \text { for } N+1 \leqslant n \leqslant 2 N-1 \text { modulo } 2 N .\end{cases}
$$

We first study the sum $\Sigma^{+}$in (3.17) collecting all terms modulo $2 N$. Then,

$$
\begin{aligned}
\Sigma^{+}=\sum_{k=0}^{\infty} \sum_{l=1}^{N-1}\left\{(l+2 k N)^{2 \alpha+2 j-1}\left|c_{l}\left(\hat{w}_{l}^{d}\right)_{l+2 k N}\right|^{2}\right. \\
\left.-(l+(2 k+1) N)^{2 \alpha+2 j-1}\left|c_{l}\left(\hat{w}_{l}^{d}\right)_{l+(2 k+1) N}\right|^{2}\right\} .
\end{aligned}
$$

By the recurrence relation (3.8) we are able to write

$$
\Sigma^{+}=\sum_{k=0}^{\infty} \sum_{l=1}^{N-1}\left\{(l+2 k N)^{2 \alpha+2 j-1}\left[1-\left(\frac{l+2 k N}{l+(2 k+1) N}\right)^{d-2 \alpha+1}\right]\left|c_{l}\left(\hat{w}_{l}^{d}\right)_{l+2 k N}\right|^{2}\right\} .
$$

Since $d-2 \alpha+1>1$, we have a lower estimate by taking only $k=0$ in (3.18), i.e.,

$$
\begin{aligned}
\Sigma^{+} & \geqslant \sum_{l=1}^{N-1} l^{2 \alpha+2 j-1}\left[1-\left(\frac{l}{l+N}\right)^{d-2 \alpha+1}\right]\left|c_{l}\left(\hat{w}_{l}^{d}\right)\right|_{l}^{2} \\
& \geqslant \kappa \sum_{l=1}^{N-1} l^{2 \alpha+2 j-1}\left|c_{l}\left(\hat{w}_{l}^{d}\right)\right|_{l}^{2}=: \kappa \sum_{0}^{+},
\end{aligned}
$$

where $\kappa=\left(1-\left(\frac{1}{2}\right)^{d-2 \alpha+1}\right)$.

In the same way we estimate the second sum $\Sigma^{-}$in (3.17) by

$$
\Sigma^{-} \geqslant \kappa \sum_{l=1}^{N-1} l^{2 \alpha+2 j-1}\left|c_{N-l}\left(\hat{w}_{N-l}^{d}\right)_{-l}\right|^{2}=: \kappa \sum_{0}^{-}
$$

On the other hand, we have for the trial function $v$ given by (3.11),

$$
\begin{aligned}
\|v\|_{\alpha+j-1 / 2}^{2} & =\left|c_{0}\right|^{2}+(2 \pi)^{2 \alpha+2 j-1} \sum_{n \neq m N}|n|^{2 \alpha+2 j-1}\left|c_{[n]}\left(\hat{w}_{[n]}^{d}\right)_{n}\right|^{2} \\
& =:\left|c_{0}\right|^{2}+(2 \pi)^{2 \alpha+2 j-1} \sum,
\end{aligned}
$$

where we find for the sum $\sum$ by (3.8)

$$
\begin{aligned}
\Sigma & =\sum_{k=0}^{\infty} \sum_{l=1}^{N-1}(l+k N)^{2 \alpha+2 j-1}\left(\frac{l}{l+k N}\right)^{2 d+2}\left\{\left|c_{l}\left(\hat{w}_{l}^{d}\right)\right|_{l}^{2}+\left|c_{N-l}\left(\hat{w}_{N-l}^{d}\right)_{-l}\right|^{2}\right\} \\
& =\sum_{l=1}^{N-1} l^{2 \alpha+2 j-1}\left[\left|c_{l}\left(\hat{w}_{l}^{d}\right)_{l}\right|^{2}+\left|c_{N-l}\left(\hat{w}_{N-l}^{d}\right)_{-l}\right|^{2}\right] \cdot \sum_{k=0}^{\infty}\left(\frac{l}{l+k N}\right)^{d-2 \alpha+1} .
\end{aligned}
$$

For every $1 \leqslant l \leqslant N-1$, we apply for an upper bound

$$
\sum_{k=0}^{\infty}\left(\frac{l}{l+k N}\right)^{d-2 \alpha+1} \leqslant \sum_{k=0}^{\infty}\left(\frac{1}{1+k}\right)^{d-2 \alpha+1}=: s<\infty,
$$

which yields from (3.22),

$$
\Sigma \leqslant s\left(\Sigma_{0}^{+}+\Sigma_{0}^{-}\right)
$$


By combining the formulae (3.17), (3.19), (3.20), (3.21) and (3.23) we achieve with the strong ellipticity $\operatorname{Re} \sigma_{ \pm}(1)>0$, the relation

$$
\left|\langle A v, \phi\rangle_{j}\right| \geqslant \operatorname{Re}\langle A v, \phi\rangle_{j} \geqslant \gamma\|v\|_{\alpha+j-1 / 2}^{2}
$$

where $\gamma=\min \left(\operatorname{Re} \sigma_{+}, \kappa s^{-1}(2 \pi)^{-2 \alpha} \operatorname{Re} \sigma_{+}, \kappa s^{-1}(2 \pi)^{-2 \alpha} \operatorname{Re} \sigma_{-}\right)$.

On the other hand, we have by (3.14), (3.16) and (3.12) for the Fourier coefficients of the test function $\phi$, the formula

$$
\left|(\hat{\phi})_{n}\right|=\frac{1}{|2 n \pi|}\left|c_{[n]}\left(\hat{w}_{[n]}^{d}\right)_{n}\right|=\frac{1}{|2 n \pi|}\left|(\hat{v})_{n}\right|, \quad n \neq 0
$$

and

$$
(\hat{\phi})_{0}=(\hat{v})_{0}
$$

It therefore follows that

$$
\|v\|_{\alpha+j-1 / 2}=\|\phi\|_{\alpha+j+1 / 2}
$$

which yields, by (3.24), the estimate

$$
\left|\langle A v, \phi\rangle_{j}\right| \geqslant \gamma\|v\|_{\alpha+j-1 / 2}\|\phi\|_{\alpha+j+1 / 2} .
$$

This implies the validity of the stability condition (3.6).

In order to derive the convergence results we now recall the following properties of the family of spline spaces (see [7, (2.1.4) ff. and (2.1.30) ff.]).

Approximation property. If $-\infty<t \leqslant s \leqslant d+1$ and $t<d+1 / 2$, there exists a constant $c$ depending only on $t, s$ and $d$, such that

$$
\inf _{\phi \in S_{d}(\Delta)}\|u-\phi\|_{t} \leqslant c h^{s-t}\|u\|_{s}, \quad u \in H^{s} .
$$

This implies for $t<d=1 / 2$,

$$
\lim _{h \rightarrow 0} \inf _{\phi \in S_{d}(\Delta)}\|u-\phi\|_{t}=0, \quad u \in H^{t} .
$$

Since the family of meshes is uniform we also have the

Inverse property.

$$
\|v\|_{0} \leqslant c h^{\tau-\sigma}\|v\|_{\tau}, \quad v \in S_{d}(\Delta)
$$

for every $\tau \leqslant \sigma<d+1 / 2$.

This will be needed to achieve the error estimates with respect to the norms higher than $j+\alpha-1 / 2$.

For the spaces $S_{d+1}(\tilde{\Delta})$ there hold the corresponding properties.

Here, and for the following proof, it is convenient to introduce the notation of the $j$-adjoint $B^{*}$ of any bounded linear operator $B: H^{s} \rightarrow H^{t}$. For any $j \in \mathbf{R}$ the $j$-adjoint $B^{*}$ is uniquely defined by

$$
\langle B u, v\rangle_{j}=\left\langle u, B^{*} v\right\rangle_{j}, \quad u \in H^{s}, v \in H^{2 j-t}
$$

and $B^{*}: H^{2 j-t} \rightarrow H^{2 j-s}$ is bounded. If $K: H^{s} \rightarrow H^{t}$ is compact, then the $j$-adjoint $K^{*}: H^{2 j-t} \rightarrow H^{2 j-s}$ is also compact.

Now we are able to show the Babuška stability conditions (3.6) also for the more general operator $A$ as given in (2.10). 
THEOREM 3.3. Let $A: H^{j+\alpha-1 / 2} \rightarrow H^{j-\alpha-1 / 2}$ be an isomorphism and

$$
A=\sigma_{+} P_{2 \alpha}^{+}+\sigma_{-} P_{2 \alpha}^{-}+K=: A_{0}+K \text { with } \operatorname{Re} \sigma_{+}, \operatorname{Re} \sigma_{-}>0 \text {, }
$$

where $K: H^{j+\alpha-1 / 2} \rightarrow H^{j-\alpha-1 / 2}$ is compact. Then there exist two positive constants $\gamma$ and $h_{0}$ such that for every $0<h \leqslant h_{0}$ the inequality (3.6) is valid.

Proof. We first note that the j-adjoint $A^{*}=A_{0}^{*}+K^{*}: H^{j+\alpha+1 / 2} \rightarrow H^{j-\alpha+1 / 2}$ is an isomorphism since it is one-to-one and has the Fredholm index zero. By the same reason one verifies that the mapping $I-\left(A^{*}\right)^{-1} K^{*}: H^{j+\alpha+1 / 2} \rightarrow H^{j+\alpha+1 / 2}$ also is an isomorphism. Let $P_{h}$ be the orthogonal projection $P_{h}: H^{j+\alpha+1 / 2} \rightarrow S_{d+1}(\tilde{\Delta})$. For a given element $v \in S_{d}(\Delta)$ there exists, by Theorem 3.2, an element $\phi \in S_{d+1}(\tilde{\Delta})$, such that

$$
\left|\left\langle A_{0} v, \phi\right\rangle_{j}\right| \geqslant \gamma^{\prime}\|v\|_{j+\alpha-1 / 2} \cdot\|\phi\|_{j+\alpha+1 / 2},
$$

where $\gamma^{\prime}>0$ is a constant independent of $v$ and $\phi\left(\right.$ e.g. $\left.\gamma^{\prime}=\gamma / 2\right)$. To $\phi$ we choose the element $\psi \in S_{d+1}(\tilde{\Delta})$ by

$$
\psi=\phi-P_{h}\left(A^{*}\right)^{-1} K^{*} \phi
$$

Then, we have

$$
\begin{aligned}
\langle A v, \psi\rangle_{j} & =\left\langle A v, \phi-\left(A^{*}\right)^{-1} K^{*} \phi\right\rangle_{j}+\left\langle A v,\left(I-P_{h}\right)\left(A^{*}\right)^{-1} K^{*} \phi\right\rangle_{j} \\
& =\left\langle A_{0} v, \phi\right\rangle_{j}+\left\langle A v,\left(I-P_{h}\right)\left(A^{*}\right)^{-1} K^{*} \phi\right\rangle_{j} .
\end{aligned}
$$

Since $\left(A^{*}\right)^{-1} K^{*}$ is a compact operator in $H^{j+\alpha+1 / 2}$, it follows from the approximation property (3.26) applied to $S_{d+1}(\tilde{\Delta})$, that

$$
\left\|\left(I-P_{h}\right)\left(A^{*}\right)^{-1} K^{*}\right\|=\varepsilon(h) \rightarrow 0 \quad \text { if } h \rightarrow 0
$$

with respect to the operator norm [13, Hilfssatz 3].

Now, (3.29), (3.30) and (3.31) yield for $0<h \leqslant h_{1}$ with a suitably chosen $h_{1}>0$,

$$
\left|\langle A v, \psi\rangle_{j}\right| \geqslant\left(\frac{\gamma^{\prime}}{2}\right)\|v\|_{j+\alpha-1 / 2}\|\phi\|_{j+\alpha+1 / 2},
$$

which in turn implies the required inequality (3.6)

$$
\left|\langle A v, \psi\rangle_{j}\right| \geqslant\left(\frac{\gamma^{\prime}}{3}\right)\|v\|_{j+\alpha-1 / 2}\|\psi\|_{j+\alpha+1 / 2}
$$

for all $0<h \leqslant h_{0}$ if $h_{0}$ is chosen small enough.

Finally, we consider the operators $A_{\Delta}:=\left(I-J+J_{\Delta}\right) A: H^{j+\alpha-1 / 2} \rightarrow H^{j-\alpha-1 / 2}$. These are uniformly bounded with respect to the discretization parameter $0<h \leqslant 1$ [7]. Thus, the sesquilinear forms

$$
\left\langle A_{\Delta} u, \phi\right\rangle_{j} \text { for }(u, \phi) \in H^{j+\alpha-1 / 2} \times H^{j-\alpha-1 / 2}
$$

are uniformly bounded. Moreover, we can show

THEOREM 3.4. Let $A: H^{j+\alpha-1 / 2} \rightarrow H^{j-\alpha-1 / 2}$ be an isomorphism as in Theorem 3.3. Then there exist two positive numbers $\gamma_{1}$ and $h_{1}$, such that for all $0<h \leqslant h_{1}$, the Babuška stability condition

$$
\inf _{\substack{l, \in S_{d(}(\Delta) \\\|v\|_{j+\alpha}(1 / 2=1}} \sup _{\substack{\phi \in S_{d+1}(\Delta) \\\|\phi\|_{j+\alpha+1 / 2}=1}}\left|\left\langle A_{\Delta} v, \phi\right\rangle_{j}\right| \geqslant \gamma_{1}
$$

is valid. 
Proof. It suffices to observe that with the trapezoidal rule

$$
\begin{aligned}
\left|\left\langle A_{\Delta} v, \phi\right\rangle_{j}\right| & \geqslant\left|\langle A v, \phi\rangle_{j}\right|-\left|\left(J-J_{\Delta}\right) A v\right|\left|\langle 1, \phi\rangle_{j}\right| \\
& \geqslant\left|\langle A v, \phi\rangle_{j}\right|-c h^{1 / 2}\|A v\|_{1 / 2}\|\phi\|_{j+\alpha+1 / 2} \\
& \geqslant\left(\gamma-c h^{1 / 2}\right)\|v\|_{j+\alpha-1 / 2} \cdot\|\phi\|_{j+\alpha+1 / 2},
\end{aligned}
$$

because of (3.3), and to apply Theorem 3.3.

As a consequence, we have the quasioptimal asymptotic convergence for the approximate solutions.

THEOREM 3.5. Let the assumptions of Theorem 3.4 be valid. If $u \in H^{j+\alpha-1 / 2}$, then the collocation equations (3.4) have a unique solution $u_{\Delta} \in S_{d}(\Delta)$ if $0<h \leqslant h_{1}$, and

$$
\left\|u-u_{\Delta}\right\|_{j+\alpha-1 / 2} \leqslant c \inf _{v \in S_{d}(\Delta)}\|u-v\|_{j+\alpha-1 / 2} \text {. }
$$

In particular, if $u \in H^{s}, j+\alpha-1 / 2 \leqslant s \leqslant d+1$, then for $j+\alpha-1 / 2 \leqslant t \leqslant s$, $t<d+1 / 2$ we find the asymptotic error estimate

$$
\left\|u-u_{\Delta}\right\|_{t} \leqslant c h^{s-t}\|u\|_{s} .
$$

Proof. The quasioptimality (3.32) follows by the previous theorem from Cea's lemma [10, Theorem 6.2.1, p. 186]. The approximation property (3.25) then implies the estimate (3.33) for $t=j+\alpha-1 / 2$. As usual, the inverse property (3.27) finally yields the statement (3.33) for the remaining values of $t$.

By using the Aubin-Nitsche duality argument we can show higher-order convergence for the error measured by the norms of lower order than $j+\alpha-1 / 2$. The proof is analogous to that of [7, Theorem 2.1.6], and therefore will be omitted. As in [7] we say that an isomorphism $B: H^{j+\alpha+1 / 2} \rightarrow H^{j-\alpha+1 / 2}$ is $s$-regular, $s>j+\alpha+$ $1 / 2$, if $B^{-1}$ maps $H^{s-2 \alpha}$ continuously onto $H^{s}$. Then, we have

TheOREM 3.6. Let $t \in[2 \alpha, j+\alpha-1 / 2]$ and assume that $A^{*}$ is $(2(j+\alpha)-t)$ regular, where $A$ is an operator as described in Theorem 3.3. In the case $t \leqslant 2 \alpha+1 / 2$, $j-\alpha-1 / 2>2$, we also assume that $A$ maps $H^{2+2 \alpha}$ boundedly into $H^{2}$. Then the Aubin-Nitsche trick provides the following superapproximation estimate:

$$
\left\|u-u_{\Delta}\right\|_{t} \leqslant \operatorname{ch}^{j+\alpha-1 / 2-t}\left\|u-u_{\Delta}\right\|_{j+\alpha-1 / 2} .
$$

Consequently, if $u \in H^{s}, j+\alpha-1 / 2 \leqslant s \leqslant d+1$, there holds

$$
\left\|u-u_{\Delta}\right\|_{t} \leqslant c h^{s-t}\|u\|_{s} \text {. }
$$

Finally, in terms of the pseudo-differential operators considered here, we have

THEOREM 3.7. Let $A$ be a strongly elliptic pseudo-differential operator with $a$ positive-homogeneous principal symbol $\sigma(\xi)$ of degree $2 \alpha$. Further, let $A: H^{j+\alpha-1 / 2} \rightarrow$ $H^{j-\alpha-1 / 2}$ be an isomorphism. Then the statements of the previous Theorems 3.5 and 3.6 are valid with $A$.

Proof. It suffices to observe that the collocation equations (3.2) are equivalent to

$$
\left(\Theta A u_{\Delta}\right)\left(t_{j}\right)=(\Theta f)\left(t_{j}\right), \quad j=1, \ldots, N,
$$

where $\Theta A$ has the representation (3.28). The regularity assumption in Theorem 3.6 is also satisfied as a consequence of the index-theorem for pseudo-differential operators on compact manifolds, [32, p. 105]. 
3.2. General Systems. In this final section we briefly show how the foregoing results can be extended to the more general systems (2.1). We consider systems where all single equations have the decomposition (2.10) with the same value $\alpha$. For the systems let $\mathscr{H}^{s}=\left(H^{s}\right)^{p} \times \mathbf{C}^{q}$ and define the bounded linear operator $\mathscr{A}$ connected with the problem (2.1) by

$$
\mathscr{A}=\left(\begin{array}{ll}
A & B \\
\Lambda & 0
\end{array}\right)
$$

$\mathscr{A}: \mathscr{H}^{s} \rightarrow \mathscr{H}^{s-2 \alpha}\left(s\right.$ to be fixed). Here $A:\left(H^{s}\right)^{p} \rightarrow\left(H^{s-2 \alpha}\right)^{p}, B: \mathbf{C}^{q} \rightarrow\left(H^{s}\right)^{p}$ and $\Lambda:\left(H^{s}\right)^{p} \rightarrow \mathbf{C}^{q}$ are bounded linear mappings. The spaces $\mathscr{H}^{s}$ present Hilbert spaces endowed with corresponding natural scalar products.

We now write the equation (2.1) in the form

$$
\mathscr{A} \mathscr{U}=\mathscr{F}
$$

where $\mathscr{U}=(u, \omega)$ and $\mathscr{F}=(f, \beta)$.

The solution $\mathscr{U} \in \mathscr{H}^{j+\alpha-1 / 2}$ will be approximated by the trial "functions" $v=$ $(v, \mu),(v, \mu) \in \mathscr{T}_{d}(\Delta):=\left(S_{d}(\Delta)\right)^{p} \times \mathbf{C}^{q}$. As in the previous section we require the restriction

$$
d>2 \alpha, \quad d \geqslant 0,
$$

and denote $j=d / 2+1$. In the Petrov-Galerkin type formulation of the collocation equations, i.e.,

$$
\begin{gathered}
\left(A u_{\Delta}\right)\left(t_{j}\right)+\left(B \omega_{\Delta}\right)\left(t_{j}\right)=f\left(t_{j}\right), \quad j=1, \ldots, N, \\
\Lambda u_{\Delta}=\beta,
\end{gathered}
$$

we use the space of test functions $\mathscr{T}_{d+1}(\tilde{\Delta})=\left(S_{d+1}(\tilde{\Delta})\right)^{p} \times \mathbf{C}^{q}$. Moreover, we denote

$$
\mathscr{A}_{\Delta}:=\left(\begin{array}{cc}
A_{\Delta} & B_{\Delta} \\
\Lambda & 0
\end{array}\right) .
$$

By Lemma 3.1 the collocation equations for $\mathscr{U} \in \mathscr{H}^{j+\alpha-1 / 2}$ are equivalent to finding $\mathscr{U}_{\Delta}=\left(u_{\Delta}, \omega_{\Delta}\right) \in \mathscr{T}_{d}(\Delta)$ such that

$$
\left\langle A_{\Delta} u_{\Delta}, \Phi\right\rangle_{j}=\left\langle\mathscr{A}_{\Delta} \mathscr{U}, \Phi\right\rangle_{j} \text { for all } \Phi \in \mathscr{T}_{d+1}(\tilde{\Delta}) \text {. }
$$

The operator $A$ can be written as

$$
A=\sigma_{+} P_{2 \alpha}^{+}+\sigma_{-} P_{2 \alpha}^{-}+K,
$$

where $K: H^{s} \rightarrow H^{s-2 \alpha}$ is compact for every $s \in \mathbf{R}$ and where $\sigma_{+}$and $\sigma_{-}$are matrices. For definiteness we shall use the requirement

$$
\operatorname{Re} \zeta^{\mathrm{T}} \boldsymbol{\sigma}_{ \pm} \bar{\zeta} \geqslant \sigma_{0}|\zeta|^{2} \text { for all } \zeta \in \mathbf{C}^{p},
$$

i.e., strong ellipticity (2.2) with $\Theta=I$, the identity. Here we have

THEOREM 3.8. Let $\mathscr{A}: \mathscr{H}^{j+\alpha-1 / 2} \rightarrow \mathscr{H}^{j-\alpha-1 / 2}$ be defined by (3.34) with $A$ having the form (3.36), where $\sigma_{+}$and $\sigma_{-}$are matrices satisfying (3.37). Moreover, we assume that $\mathscr{A}$ is an isomorphism. Then, there exist positive numbers $\gamma_{0}$ and $h_{0}$ such that for all $0<h \leqslant h_{0}$ the Babuška stability condition $\left(\mathscr{A}_{*}=\mathscr{A}_{\left.\text {or } \mathscr{A}_{\Delta}\right)}\right.$

$$
\inf _{\substack{\nu \in \mathscr{T}_{d}(\Delta) \\\|\nu\|_{j+\alpha-1 / 2}=1}} \sup _{\substack{\Phi \in \mathscr{T}_{d+1}(\tilde{\Delta}) \\\|\Phi\|_{j+\alpha+1 / 2}=1}}\left|\left\langle\mathscr{A}_{*} \nu, \Phi\right\rangle_{j}\right| \geqslant \gamma_{0}
$$

is valid. 
Proof. If we handle compact and small perturbations as in the previous section, it suffices to consider the form

$$
\langle A v, \phi\rangle_{j}, \quad v \in\left(S_{d}(\Delta)\right)^{p}, \quad \phi \in\left(S_{d+1}(\tilde{\Delta})\right)^{p},
$$

where the operator

$$
A=\sigma_{+} P_{2 \alpha}^{+}+\sigma_{-} P_{2 \alpha}^{-}
$$

is defined componentwise in any obvious way. We define the mapping $Q:\left(S_{d}(\Delta)\right)^{p}$ $\rightarrow\left(S_{d+1}(\tilde{\Delta})\right)^{p}$ such that for a given element $v \in\left(S_{d}(\Delta)\right)^{p}$ the components of $Q v$ are defined by the rules (3.13) and (3.16) with the matrix decomposition $\sigma_{+}=\operatorname{Re} \sigma_{+}+$ $i \operatorname{Im} \sigma_{+}$, where

$$
\operatorname{Re} \sigma_{+}=\frac{1}{2}\left(\sigma_{+}+\left(\sigma_{+}\right)^{*}\right) \text { and } \operatorname{Im} \sigma_{+}=\frac{1}{2 i}\left(\sigma_{+}-\left(\sigma_{+}\right)^{*}\right) ;
$$

we have

$$
\left\langle\sigma_{+} P_{2 \alpha}^{+} v, Q v\right\rangle_{j}=\left\langle\operatorname{Re} \sigma_{+} P_{2 \alpha}^{+} v, Q v\right\rangle_{j}+i\left\langle\operatorname{Im} \sigma_{+} P_{2 \alpha}^{+} v, Q v\right\rangle_{j} .
$$

One verifies that

$$
\left\langle P_{2 \alpha}^{+} u, Q v\right\rangle_{j}=\overline{\left\langle P_{2 \alpha}^{+} v, Q u\right\rangle_{j}}
$$

for all $u \in H^{j+\alpha-1 / 2}$ and $v \in H^{j+\alpha+1 / 2}$ (where $Q$ is understood as scalar-valued). By using (3.40) we find that the last term in (3.39) is purely imaginary. The positive-definiteness (3.37) allows the representation $\operatorname{Re} \sigma_{+}=\Gamma_{+}^{*} \Gamma_{+}$with some regular invertible matrix $\Gamma_{+}$. Hence, we obtain from (3.39),

$$
\begin{aligned}
\operatorname{Re}\left\langle\sigma_{+} P_{2 \alpha}^{+} v, Q v\right\rangle_{j} & =\operatorname{Re}\left\langle\Gamma_{+}^{*} \Gamma_{+} P_{2 \alpha}^{+} v, Q v\right\rangle_{j}=\operatorname{Re}\left\langle P_{2 \alpha}^{+} \Gamma_{+} v, Q \Gamma_{+} v\right\rangle_{j} \\
& =\sum_{k=1}^{p} \operatorname{Re}\left\langle P_{2 \alpha}^{+}\left(\Gamma_{+} v\right)_{k}, Q\left(\Gamma_{+} v\right)_{k}\right\rangle_{j} .
\end{aligned}
$$

Recalling the proof of Theorem 3.2, we find the lower bound

$$
\begin{aligned}
\operatorname{Re}\left\langle\sigma_{+} P_{2 \alpha}^{+} v, Q v\right\rangle_{j} & \geqslant \gamma \sum_{k=1}^{p}\left\|P_{2 \alpha}^{+}\left(\Gamma_{+} v\right)_{k}\right\|_{j+\alpha-1 / 2}^{2} \\
& \geqslant \gamma+\sum_{k=1}^{p}\left\|\left(P_{2 \alpha}^{+} v\right)_{k}\right\|_{j+\alpha-1 / 2}^{2} .
\end{aligned}
$$

Similarly, we find

$$
\operatorname{Re}\left\langle\sigma_{-} P_{2 \alpha}^{-} v, Q v\right\rangle_{j} \geqslant \gamma_{-} \sum_{k=1}^{p}\left\|\left(P_{2 \alpha}^{-} v\right)_{k}\right\|_{j+\alpha-1 / 2}^{2},
$$

and accordingly with (3.38), (3.41) and (3.42),

$$
\operatorname{Re}\langle A v, Q v\rangle_{j} \geqslant \gamma\|v\|_{j+\alpha-1 / 2}^{2} \geqslant \gamma_{1}\|v\|_{j+\alpha-1 / 2}\|Q v\|_{j+\alpha+1 / 2},
$$

which yields the required assertion.

Similarly as in the previous section the stability result of Theorem 3.8 together with the approximation and inverse properties provide the following convergence result. 
THEOREM 3.9. Let the assumptions of Theorem 3.8 be valid. If $u \in\left(H^{j+\alpha-1 / 2}\right)^{p}$, then the collocation equations (3.35) have a unique solution $\mathscr{U}_{\Delta} \in \mathscr{T}_{d}(\Delta)$ for $0<h \leqslant h_{0}$, and we have

$$
\left\|u-u_{\Delta}\right\|_{j+\alpha-1 / 2}+\left|\omega-\omega_{\Delta}\right| \leqslant c \inf _{v \in S_{d}(\Delta)}\|u-v\|_{j+\alpha-1 / 2} .
$$

If, in addition, $u \in\left(H^{s}\right)^{p}, j+\alpha-1 / 2 \leqslant s \leqslant d+1$, then the asymptotic error estimates

$$
\left\|u-u_{\Delta}\right\|_{t}+\left|\omega-\omega_{\Delta}\right| \leqslant c h^{s-t}\|u\|_{s}
$$

are true for $j+\alpha-1 / 2 \leqslant t \leqslant s$. If $t \in[2 \alpha, j+\alpha-1 / 2]$ and if $\mathscr{A}^{*}$ is $(2(j+\alpha)-$ $t$ )-regular and if in the case $t \leqslant 2 \alpha+1 / 2, j-\alpha-1 / 2>2$, $\mathscr{A}$ maps $\mathscr{H}^{2+2 \alpha}$ boundedly into $\mathscr{H}^{2}$, then we obtain

$$
\left\|u-u_{\Delta}\right\|_{t}+\left|\omega-\omega_{\Delta}\right| \leqslant c h^{j+\alpha-1 / 2-t}\left\|u-u_{\Delta}\right\|_{j+\alpha-1 / 2} .
$$

With the inverse assumption, (3.44) holds again for $[2 \alpha \leqslant t \leqslant j+\alpha-1 / 2]$ if $u \in$ $\left(H^{2}\right)^{p}, j+\alpha-1 / 2 \leqslant s \leqslant d+1$.

The definition of the $j$-adjoint $\mathscr{A}^{*}$ and the notion of $s$-regularity in case of the system are obvious.

Finally, since the collocation equations (3.35) and the equations

$$
\begin{aligned}
\Theta\left(A u_{\Delta}\right)\left(t_{j}\right)+\Theta B\left(t_{j}\right) \omega_{\Delta} & =\Theta f\left(t_{j}\right), \quad j=1, \ldots, N, \\
\Lambda u_{\Delta} & =\beta
\end{aligned}
$$

are equivalent, we have corresponding to Theorem 3.7:

THEOREM 3.10. Let $A$ be a strongly elliptic system of pseudo-differential operators with a positive-homogeneous principal symbol $\sigma(\xi)$ of degree $2 \alpha$. Further, let $\mathscr{A}$ : $\mathscr{H}^{j+\alpha-1 / 2} \rightarrow \mathscr{H}^{j-\alpha-1 / 2}$ be an isomorphism. For the lower-order estimates (3.44) with $t \in[2 \alpha, j+\alpha-1 / 2]$ we require in addition that $\mathscr{A}: \mathscr{H}^{t} \rightarrow \mathscr{H}^{t-2}$ is injective. Then the statements of the previous Theorem 3.9 are all valid.

\footnotetext{
Department of Mathematics

Faculty of Technology

University of Oulu

Linnanmaa

90570 Oulu 57, Finland

Department of Mathematics

Technische Hochschule Darmstadt

Schlossgartenstr. 7

D-1600 Darmstadt

Federal Republic of Germany
}

1. M. S. AвоU EL-SEOUd, Numerische Behandlung von schwach singulären Integralgleichungen erster Art, Doctoral Dissertation. Technische Hochschule Darmstadt, Germany, 1979.

2. M. S. ABOU EL-SEOUD, "Kollokationsmethode für schwach singuläre Instegralgleichungen erster Art," Z. Angew. Math. Mech., v. 59, 1979, pp. T45-T47.

3. M. S. AGRANOvich, "Elliptic singular integro-differential operators," Russian Math. Surveys, v. 20, Nos. 5/6, 1965, pp. 1-122.

4. M. S. AgRANOvich, "Spectral properties of elliptic pseudodifferential oeprators on a closed curve," Functional Anal. Appl., v. 13, 1979, pp. 279-281.

5. M. A. Alexsidze, The Solution of Boundary Value Problems with the Method of the Expansion with Respect to Nonorthonormal Functions, "Nauka”, Moscow, 1978. (Russian) 
6. P. Anselone, Collectively Compact Operator Theory, Prentice-Hall, London, 1971.

7. D. N. ARNold \& W. L. Wendland, "On the asymptotic convergence of collocation methods," Math. Comp., v. 41, 1983, pp. 349-381.

8. K. E. Atkinson, A Survey of Numerical Methods for the Solution of Fredholm Integral Equations of the Second Kind, SIAM, Philadelphia, Pa., 1976.

9. J. P. Aubin, Approximation of Elliptic Boundary Value Problems, Wiley, New York, 1972.

10. I. BABUSKA \& A. K. AzIz, "Survey lectures on the mathematical foundations of the finite element method," The Mathematical Foundation of the Finite Element Method with Applications to Partial Differential Equations (A. K. Aziz, ed.), Academic Press, New York, 1972, pp. 3-359.

11. C. BAKER, The Numerical Treatment of Integral Equations, Clarendon Press, Oxford, 1977.

12. J. BluE, "Boundary integral solution of Laplace's equations," Bell System Tech. J., v. 57, 1978, pp. 2797-2822.

13. G. BRUhn \& W. L. Wendland, "Über die näherungsweise Lösung von linearen Funktionalgleichungen," Funktionalanalysis, Approximationstheorie, Numerische Mathematik (L. Collatz, ed.), Intern. Ser. Num. Math., v. 7, Birkhäuser, Basel, 1967, pp. 136-144.

14. S. Christiansen, "Numerical solutions of an integral equation with a logarithmic kernel," $B I T, v$. 11,1971 , pp. 276-287.

15. M. DJaOua, "A method of calculation of lifting flows around 2-dimensional corner shaped bodies," Math. Comp., v. 36, 1981, pp. 405-425.

16. M. DuRAND, "Layer potential and boundary value problems for the Helmholtz equation in the complement of a thin obstacle," Math. Methods Appl. Sci., v. 5, 1983, pp. 389-421.

17. P. J. T. FilipPI, "Layer potentials and acoustic diffraction," Sound Vibration, v. 54, 1977, pp. $473-500$.

18. J. Giroire \& J. C. Nedelec. "Numerical solution of an exterior Neumann problem using a double layer potential," Math. Comp., v. 25, 1978, pp. 973-990.

19. J. K. HAyes, D. K. Kahaner \& R. G. Kellner, “An improved method for numerical conformal mapping," Math. Comp., v. 26, 1972, pp. 327-334.

20. G. C. HSiaO, P. Kopp \& W. L. Wendland, "A Galerkin collocation method for some integral equations of the first kind," Computing, v. 25, 1980, pp. 89-130.

21. G. C. Hsiao \& W. L. Wendland, "A finite element method for some integral equations of the first kind," J. Math. Anal. Appl., v. 58, 1977, pp. 449-481.

22. G. C. Hsiao \& W. L. Wendland, “The Aubin-Nitsche lemma for integral equations," J. Integral Equations, v. 3, 1981, pp. 299-315.

23. J. L. Lions \& E. Magenes, Non-Homogeneous Boundary Value Problems and Applications I, Springer-Verlag, Berlin and New York, 1972.

24. S. G. Michlin \& S. Prössdorf, Singuläre Integraloperatoren, Akademie-Verlag, Berlin, 1980.

25. N. I. MUSKHElishvili, Singular Integral Equations, Noordhoff, Groningen, 1953.

26. G. G. Mustoe \& I. C. Mathews, Direct Boundary Integral Methods, Point Collocation and Variational Procedures, Preprint, Univ. Coll. Swansea, U.K., 1982.

27. P. M. Prenter, "A collocation method for the numerical solution of integral equations," SIAM J. Numer. Anal., v. 10, 1973, pp. 570-581.

28. J. SaRANEN \& W. L. WENDland, "The Fourier series representation of pseudo-differential operators on closed curves," Complex Variables. (To appear.)

29. (j. SснміDт, “On spline collocation for singular integral equations," Math. Nachr., v. 111, 1983, pp. $177-196$.

30. R. Seeley, “Topics in pseudo-differential operators," Pseudo-Differential Operators (L. Nirenberg, ed.), CIME, Cremonese, Roma, 1969, pp. 169-305.

31. G. T. Symm, "Integral equation methods in potential theory II," Proc. Roy. Soc. London Ser. A, v. 275, 1963, pp. 33-46.

32. F. Trèves, Introduction to Pseudodifferential and Fourier Integral Operators I, Plenum Press, New York and London, 1980.

33. V. V. Voronin \& V. A. Сесоно, "An interpolation method for the solution of an integral equation of the first kind with a logarithmic singularity," Dokl. Akad. Nauk SSSR, v. 216, 1974, pp. 1209-1211; English transl. in Soviet Math. Dokl., v. 15, 1974, pp. 949-952.

34. J. O. WATSON, "Advanced implementation of the boundary element method for two- and three-dimensional elastostatics," Developments in Boundary Element Methods-1 (P. K. Banerjee and R. Butterfield, eds.), Appl. Sci. Publ. TLD, London, 1979, pp. 31-63.

35. W. L. WENDLAND, "Boundary element methods and their asymptotic convergence," Theoretical A coustics and Numerical Techniques (P. Filippi, ed.), CISM Courses and Lectures No. 277, Springer-Verlag, Wien, New York, 1983, pp. 135-216. 